Revue internationale P.M.E.

Économie et gestion de la petite et moyenne entreprise

\title{
Les P.M.E. face à la gestion de leurs effectifs : comment adapter les ressources humaines aux impératifs stratégiques ?
}

\section{Gérald Naro}

Volume 3, numéro 1, 1990

URI : https://id.erudit.org/iderudit/1007946ar

DOI : https://doi.org/10.7202/1007946ar

Aller au sommaire du numéro

Éditeur(s)

Presses de l'Université du Québec

ISSN

0776-5436 (imprimé)

1918-9699 (numérique)

Découvrir la revue

Citer cet article

Naro, G. (1990). Les P.M.E. face à la gestion de leurs effectifs : comment adapter les ressources humaines aux impératifs stratégiques? Revue internationale P.M.E., 3(1), 57-74. https://doi.org/10.7202/1007946ar
Résumé de l'article

Cet article repose sur l'idée qu'il existe bien une pratique effective de la gestion de l'emploi dans les P.M.E. : celle-ci se présente de façon contingente, s'inscrit dans la logique personnelle du chef d'entreprise et obéit à ses propres contraintes stratégiques.

Une telle observation met en exergue la nécessité d'adopter une pratique stratégique de la gestion des effectifs en P.M.E., cohérente avec les aspirations personnelles du dirigeant d'une part, en osmose d'autre part, avec les orientations stratégiques de base de la firme (avantage compétitif par les coûts ou par les services) compte-tenu des contraintes et incertitudes de l'environnement (état de dépendance et de vulnérabilité de la firme dans son environnement concurrentiel). 


\title{
Les P.M.E. face à la gestion de leurs effectifs : comment adapter les ressources humaines aux impératifs stratégiques?
}

\author{
Gérald NARO' \\ ERFI, Université de Montpellier I
}

\begin{abstract}
RÉSUMÉ
Cet article repose sur l'idée qu'il existe bien une pratique effective de la gestion de l'emploi dans les P.M.E. : celle-ci se présente de façon contingente, s'inscrit dans la logique personnelle du chef d'entreprise et obéit à ses propres contraintes stratégiques.

Une telle observation met en exergue la nécessité d'adopter une pratique stratégique de la gestion des effectifs en P.M.E., cohérente avec les aspirations personnelles du dirigeant d'une part, en osmose d'autre part, avec les orientations stratégiques de base de la firme (avantage compétitif par les coûts ou par les services) compte-tenu des contraintes et incertitudes de l'environnement (état de dépendance et de vulnérabilité de la firme dans son environnement concurrentiel).
\end{abstract}

\begin{abstract}
This article is based upon the idea that there does exist a practice of management of manpower in Small and Medium Sized Firms : it appears dependent, inscribes itself in the personal logic of the manager, and obeys proper strategic constraints.

Such observation emphasizes the necessity of adopting a strategic practice of management of employment in Small and Medium-sized businesses, coherent with the personal aims of the firm manager on the one hand; in osmosis on the other hand, with the basic strategic orientations of the firm (competitive advantage by the costs on by the services), taking into account the constraints and uncertainty of the environment (state of vulnerability-dependence of the firm in its competitive environment).
\end{abstract}

- Gérald Naro, agrégé d'économie et Gestion et Docteur en Sciences de gestion, est membre de l'ERFI (groupe GRH) et est Maitre de conférences de Sciences de gestion à l'Université de Montpellier 1. Il est auteur d'une thèse sur les facteurs et comportements d'embauche dans les P.M.E. (Montpellier, février 1989). Adresse : Equipe de Recherche sur la Firme et l'Industrie, Institut Supérieur de l'Entreprise de Montpellier, 14, rue Cardinal de Cabrières, 34000 Montpellier, tél. : 67615473, téléfax : 67604231. 


\section{RESUMEN}

Este articulo se funda sobre la idea que existe una pratica efectiva de la gestion del empleo en pequenas y medias empresas : esta se prosenta de manera contingencia y se inscribe en la logica personal del director de empresa obedeciendo a sus propios apremios strategicos.

Una tal observacion revela la necesidad de adoptar une pratica strategica de la gestion del empleo en paquenas y medias empresas coherencia de una parte con los objetivos personales del jete de emprasa, en osmosis de otra parte, son las orientaciones strategicas de base de la firma (ventaja competitiva por lors costes o por los servicios) teniendo en cuanta de los apremios y incertitudes externos (estado de vulnerabilidad-dependencia de la firma en su situacion competitiva). 


\section{Introduction}

Au-delà de son seul intérêt macro-économique et des espoirs dont elle est porteuse en matière de lutte contre le sous-emploi, l'observation de la part grandissante prise par les P.M.E. dans l'économie (Didier, 1982, Julien, Marenne et Naro, 1988, Laroche, 1989) et de leur contribution plus favorable à l'emploi (Birch, 1979, Birch et Mc Cracken, 1981, Story et Robinson, 1980, Storey et Johnson, 1986, Birley, 1986...), ne manque pas d'interpeller les observateurs comme les acteurs de la gestion des firmes de petite et moyenne dimensions. C'est qu'en réalité un tel phénomène affecte un domaine relativement mal connu et particulièrement sensible du management de ces entreprises : leur gestion des ressources humaines.

En premier lieu, la création d'un nombre de plus en plus important de petites entreprises dans l'économie fait apparaître en la matière de réels problèmes : dès la création, mais surtout au cours de leur phase de démarrage, lors de leur montée en compétitivité, va se poser pour ces firmes la question fondamentale de l'engagement de ressources. Dès lors, l'entrepreneur se trouve confronté à diverses interrogations d'une importance décisive :

- Parmi les ressources à mettre en œuvre, quelle doit être la part des ressources humaines? Doit-on systématiquement embaucher ou, au contraire, n'a-t-on pas intérêt à externaliser une partie des services que pourrait rendre la maind'œuvre?

- Ne va-t-il pas se poser des problèmes humains auxquels le dirigeant n'est pas préparé ? Un effectif trop élevé ne risque-t-il pas de remettre en question ses aspirations à l'autonomie ou à la convivialité ?

- Une croissance pléthorique des effectifs ne va-t-elle pas entraîner des problèmes de trésorerie à court terme?

- Par rapport aux orientations stratégiques de base et aux contraintes de l'environnement, qu'est-ce qui sera déterminant dans la gestion des ressources humaines ? Leur coût ? Leur flexibilité ? leur qualité en termes de motivation, d'adaptabilité et de disponibilité face aux besoins plus ou moins changeants et complexes de la clientèle?

Autant de questions pour un dirigeant de P.M.E. lors de la phase de démarrage. Mais le dirigeant risque fort de les rencontrer tout au long du cycle de vie de l'entreprise, notamment chaque fois que des changements de cap stratégiques, ou que de nouvelles opportunités de croissance le conduiront à mobiliser de nouvelles ressources; chaque fois également, qu'il percevra une modification des contraintes de son environnement...

En second lieu, le fait que les P.M.E. emploient une part de plus en plus importante de la population active, en révélant des aspects et des problèmes d'un nouvel ordre, nous amène à nous demander dans quelles conditions la gestion des ressources humaines tendra désormais à se réaliser : celle-ci ne va-t-elle pas exiger 
une pratique spécifique ? De nouveaux modèles de gestion du personnel, adaptés aux caractéristiques particulières des P.M.E., ne restent-ils pas à définir ?

Or sur cette question, les ouvrages et manuels de gestion du personnel ne nous renseignent guère, car ils sont dans l'ensemble fondés sur un postulat selon lequel la gestion des ressources humaines demeure l'apanage des grandes organisations (Mahé De Boislandelle, 1989). Pourtant, à l'examen, il apparaît qu'en ce domaine, les dirigeants de P.M.E. sont confrontés à des difficultés bien réelles et notamment, s'il est un aspect fondamental de leur gestion du personnel, c'est bien celui de la gestion de l'emploi.

Mais là encore, nous nous trouvons face à un champ d'investigation encore relativement peu exploré par les spécialistes des sciences de gestion. Ces derniers ont-ils considéré que le sujet ne méritait pas que l'on s'y consacre - ces problèmes étant le domaine réservé des grandes organisations - ou tout simplement, estimaientils implicitement qu'il ne présentait aucune spécificité par rapport à tout ce qu'ils écrivaient sur sa pratique dans les organisations de grande dimension (ce qui est vrai pour les grandes entreprises, l'est aussi pour les petites) ? Toujours est-il que la plupart des chercheurs en gestion des ressources humaines se sont longtemps désintéressés des problèmes de gestion des effectifs en P.M.E.

En fait, ce manque d'intérêt nous interpelle sur une question essentielle : existe-t-il en réalité une pratique effective de la gestion de l'emploi en P.M.E. ?

Dans la mesure où ces firmes dans l'ensemble, ne disposent pas de structures formelles de gestion du personnel chargées de diriger, animer, prévoir et organiser l'adéquation entre les besoins et les ressources en hommes (Gauthier et Pélissé, 1985, Mahé de Boislandelle, 1988), on est en droit de se demander si leur gestion de l'emploi ne se limite pas en définitive à une simple administration des effectifs (tenue du registre des effectifs, rédaction et signature des contrats de travail, etc,...), fonction qui dans la plupart des cas serait dévolue au comptable de l'entreprise.

En outre, quand on connaît l'extrême personnalisation qui caractérise le management des P.M.E., peut-on réellement parler de gestion de l'emploi dans la mesure où celle-ci reste fortement empreinte des logiques personnelles du dirigeant qui ne relèvent pas forcément d'une rationalité économique?

Enfin, peut-on réellement gérer l'emploi, et avec quelle marge d'autonomie, dans des entreprises qui, bien souvent, sont fortement soumises de l'extérieur, par des relations de dépendance envers des firmes de plus grande taille (Faure, Marchesnay et Mathie, 1979) qui tendent à leur imposer des contraintes de coût et de flexibilité pouvant se répercuter sur leur gestion des ressources humaines?

Comme l'on peut s'en rendre compte, les choix en matière de gestion de l'emploi dans les firmes de petite et moyenne dimensions, de par notamment leur importance décisive, la diversité des facteurs qui interfêrent dans la prise de décision, ou encore leur incidence sur l'avenir à moyen et long terme de la firme, revêtent un 
caractère stratégique indéniable et soulignent tout l'intérêt de développer une pratique stratégique de la gestion de l'emploi dans les P.M.E.

Cet article se propose d'apporter des éléments de réponse aux diverses questions soulevées par la problématique de la gestion des effectifs en P.M.E. à la lumière de conclusions et de réflexions nées de diverses enquêtes réalisées en Languedoc-Roussillon dans le cadre d'une recherche sur les facteurs et comportements d'embauche dans les P.M.E.. Nous tenterons de développer l'argument selon lequel il existe une pratique effective de la gestion de l'emploi en P.M.E., même si celle-ci ne relève pas toujours d'une politique clairement explicitée et s'inscrit dans la logique personnelle du dirigeant. Il s'agira alors de souligner toute la spécificité d'une telle pratique dans le contexte particulier des firmes de petite et moyenne dimensions, et de démontrer l'intérêt de développer une pratique stratégique de la gestion de l'emploi dans les entreprises petites et moyennes.

\section{Le contexte particulier de la gestion des effectifs en P.M.E.}

Comme l'on pourrait s'y attendre, l'extrême hétérogénéité qui caractérise l'univers des P.M.E. fait que ces firmes n'adoptent pas un mode universel de comportement en matière de gestion des effectifs, mais se caractérisent plutôt par une grande variété de pratiques. Cette contingence dans les politiques de gestion de l'emploi pourrait s'expliquer en grande partie par la diversité des profils de chefs d'entreprise que l'on peut rencontrer dans les firmes de petite et moyenne dimensions. Car l'un des traits distinctifs majeurs de ces entreprises réside dans l'extrême personnalisation de leur management : la logique personnelle du dirigeant de P.M.E. et ses aspirations fondamentales conditionnent largement ses conduites et, tout particulièrement pour ce qui nous intéresse, sa politique de gestion de l'emploi. Un autre élément d'explication tient dans les contraintes et impératifs stratégiques propres aux P.M.E. Dépendance-vulnérabilité de ces firmes dans leur environnement concurrentiel, type d'avantage compétitif recherché : autant de variables qui pourront s'avérer déterminantes dans le choix d'une politique de gestion des effectifs.

\subsection{Une pratique réelle de la gestion des effectifs...}

Il existe bien une pratique de la gestion des effectifs au sein des P.M.E. Certes, celle-ci ne revêt pas un caractère formel : il n'existe pas, comme dans les grandes organisations, une structure chargée d'analyser, de prévoir et de planifier l'adéquation des besoins et des ressources humaines. Dans les petites et moyennes organisations que nous avons étudiées, c'est en général le dirigeant qui assure ces fonctions : c'est lui qui perçoit un besoin en ressources nouvelles, c'est lui qui décide de l'opportunité d'une embauche, c'est encore lui qui, parfois assisté d'un chef de service, conduit le recrutement à son terme. Une telle pratique n'est guère souvent 
explicitée clairement : «tout se passe dans la tête du chef d'entreprise». Par exemple, l'un des patrons interrogés lors de l'enquête sur les facteurs déterminants de l'embauche, nous a expliqué qu'il venait d'embaucher un jeune ouvrier qualifié, en prévoyant que dans quatre ans ce jeune salarié pourrait assurer les fonctions d'un des contremaîtres de l'entreprise exerçant son droit à la retraite. Enfin, il est apparu clairement que la plupart des chefs de P.M.E. opèrent intuitivement une distinction entre gestion à court terme et gestion à moyen et long terme des effectifs et adaptent en conséquence leurs ressources humaines par des moyens adéquats. Il existerait ainsi dans les P.M.E. des stratégies émergentes de gestion de l'emploi.

\subsection{Une absence d'unicité dans les comportements...}

Les diverses enquêtes l'ont montré : la gestion de l'emploi en P.M.E. ne relève pas d'un modèle unique de comportement. Si nous avons pu observer des tendances lourdes (un recours croissant à l'externalisation de l'emploi, une faiblesse relative des cas de substitution de ressources techniques aux ressources humaines, l'utilisation privilégiée du contrat à durée déterminée et des heures supplémentaires comme moyen d'ajustement à court terme,...), une analyse plus approfondie des pratiques mises en œuvre dans les P.M.E. observées, témoigne de pratiques contingentes.

Au total, si l'on souhaite classer les comportements observés à l'intérieur d'une typologie, quatre groupes apparaissent :

- des P.M.E. caractérisées par une gestion de l' emploi que l'on pourrait qualifier «d'opportuniste», en ce sens qu'elle vise à adapter l'évolution des effectifs aux opportunités et contraintes de l'environnement, ces derniers pouvant évoluer à la hausse ou à la baisse selon les exigences du moment. Dans ce groupe, on observe des comportements «expansionnistes» caractérisés par un accroissement soutenu des effectifs, étant le fait d'entreprises en croissance, ainsi que des pratiques «de retrait» marquées par des licenciements et une tendance à remplacer le travail par le capital et à l'externalisation.

C'est dans ce groupe, composé en majorité des plus grosses P.M.E. de l'échantillon, que l'on constate l'éventail le plus large des moyens mis en œuvre;

- des P.M.E. dont la gestion de l'emploi se distingue par une recherche systématique d'évitement de l' embauche : recours à la sous-traitance, externalisation de services. Dans l'ensemble, il s'agissait de petites entreprises, souvent en démarrage;

- des P.M.E. dont la gestion de l'emploi reste conditionnée par le franchissement d' un seuil d' effectif à partir duquel le dirigeant va s'efforcer de stopper l'augmentation des ressources humaines, soit en poursuivant la croissance des affaires par une politique d'externalisation (dans ce cas l'on retrouve le groupe précédent), soit en autolimitant l'élévation du volume d'affaires. Trois types de seuils 
sont alors apparus comme déterminants : des seuils d'efficience, d'origine psychosociologique, à partir desquels les buts du dirigeant (autonomie, convivialité, stabilité, notamment) semblent remis en cause par l'augmentation des effectifs. Des seuils d' efficacité, d'origine technico-économique, au-delà desquels le rapport résultats/moyens engagés présente une évolution défavorable. Enfin des seuils sociaux ou fiscaux, d'origine réglementaire, dont le franchissement entraîne pour le dirigeant un certain nombre de contraintes sociales et financières. Il est à noter que ce type de comportement pouvait être observé plus particulièrement dans les P.M.E. familiales.

- des P.M.E. enfin, dont la gestion de l' emploi est caractérisée par un statut quo (refus de toute embauche nouvelle, stabilisation du niveau de l'effectif). Il s'agit alors de petites entreprises artisanales ou industrielles, ainsi que de petits commerces.

En fait, deux ensembles de facteurs afférents aux caractéristiques organisationnelles et stratégiques des P.M.E. semblent à même d'expliquer ces différences de comportements et exercer ainsi un rôle discriminant.

- En premier lieu, il s'agit des aspirations fondamentales de dirigeant et plus particulièrement de la politique de croissance qu'il entend poursuivre : c'est là en effet, un trait distinctif majeur des P.M.E., les buts du dirigeant constituent une variable clé de la conduite de la firme, et il ne faut pas s'étonner que les politiques adoptées en matière de gestion de l'emploi restent fortement empreintes des logiques personnelles du chef d'entreprise;

- En second lieu, la façon dont la P.M.E. s'insère dans son environnement concurrentiel, en termes de dépendance-vulnérabilité et d'avantages compétitifs recherchés, entraîne des contraintes et impératifs stratégiques propres qui vont déterminer sa politique de gestion des effectifs.

Dans ces conditions, on perçoit intuitivement l'intérêt d'une pratique stratégique de la gestion de l'emploi, cohérente d'une part avec les aspirations fondamentales du dirigeant en termes de croissance, intégrée d'autre part aux orientations stratégiques de base de la firme, compte tenu des conditions d'incertitude et du degré de contrainte imposés par l'environnement concurrentiel de la firme.

\section{Pour une gestion stratégique de l'emploi en P.M.E.}

Nombreux sont les auteurs qui soulignent la nécessité d'intégrer la gestion des ressources humaines dans l'analyse et la planification stratégique de la firme (Alpander, 1989, Besseyre des Horts, 1988, Miller, 1985, Quin Mills, 1986...). Notre propos dans les lignes qui suivent sera de voir comment, à l'intérieur du sous-système de gestion des ressources humaines, les décisions en matière de gestion de l'emploi participent au système général de prise de décisions dans le cadre de la gestion stratégique de la P.M.E.. 


\subsection{Fondements et objectifs d'une gestion stratégique de l'emploi en P.M.E.}

Notre travail repose sur l'idée que la gestion de l'emploi à moyen et long terme s'inscrit dans le cadre plus général du management stratégique de la firme et, à ce titre, relève des politiques adoptées en matière d'engagement de ressources (humaines, financières, physiques, d'information). Dès lors, les décisions relatives à l'engagement (ou au désengagement) de ressources humaines étant soumises aux choix plus globaux d'allocation de ressources, peuvent se voir préférer d'autres solutions. La figure $\mathrm{n}^{\circ} 1$ ci-dessous, illustre ce processus décisionnel :

Fig. 1 Les choix en matière d'engagement de ressources

\begin{tabular}{|c|c|c|c|}
\hline & ENGAGEMENT & & HUMAINES \\
\hline et/ou & DÉSENGAGEMENT & de ressources & PHYSIQUES \\
\hline et/ou & SUBSTITUTION & & FINANCIERES \\
\hline et/ou & ÉVITEMENT & & D'INFORMATION \\
\hline
\end{tabular}

Liéeà la politique d'engagement de ressources qui sera décidée dans le cadre des orientations stratégiques de base, la gestion de l'emploi se doit d'être cohérente avec ces mêmes orientations, compte tenu des contraintes organisationnelles et stratégiques qui s'exercent sur la firme.

Dans les P.M.E., une première contrainte à considérer tient dans les buts que poursuit le dirigeant : celui-ci recherche-t-il l'indépendance, la puissance, la pérennité ou la convivialité ? Selon les aspirations dominantes dans le «panier de buts» (Marchesnay, 1986), les politiques en matière d'engagement de ressources pourront s'avérer totalement différentes.

Un deuxième type de contrainte est lié à la position qu'occupe la P.M.E., dans son environnement : à son degré de vulnérabilité (en termes de turbulence, accessibilité, complexité technologiques ou commerciales) et tout particulièrement à la nature des relations qu'elle entretient avec ses principaux partenaires (clients, fournisseurs, donneurs d'ordre, sous-traitants,...). On peut alors penser que selon le degré de contrainte ou d'incertitude qui va s'exercer sur la firme du fait de sa position de dépendance et/ou de vulnérabilité, les choix qui seront réalisés en matière d'allocation des diverses ressources pourront apparaître sous des formes nettement différenciées : leur coût et leur flexibilité pourront alors apparaitre comme des variables déterminantes. 
Enfin, une gestion stratégique de l' emploi en P.M.E. passera par la prise de décisions cohérentes avec les orientations stratégiques de base de la firme.

A ce sujet, il apparaît que la stratégie des firmes de petite et moyenne dimensions passe bien souvent par le positionnement sur un segment cible, une «niche» dans laquelle elle va s'efforcer d'obtenir et de maintenir un avantage concurrentiel, soit à partir de coûts plus faibles que ses concurrents, soit grâce à une différenciation fondée notamment sur un meilleur service offert à la clientèle (Marchesnay, 1988). On retrouve ici la stratégie de concentration mentionnée notamment par M. Porter (1986), celle-ci présentant deux variantes comme l'indique la figure $n^{\circ} 2$.

Selon la nature de l'avantage concurrentiel recherché par la P.M.E. lors de sa montée en compétitivité, les choix en matière d'engagement de ressources vont s'avérer déterminants. Au niveau des ressources humaines, l'un des objectifs premiers de la gestion de l'emploi sera précisément de permettre une adéquation efficiente entre, d'une part, les besoins en ressources humaines nés de l'orientation stratégique poursuivie et, d'autre part, les ressources humaines à mobiliser. Divers moyens sont alors à la disposition du décideur.

\subsection{Les moyens à la disposition du décideur : pour une réflexion en termes d' «Emploi-Mix»}

En nous inspirant des concepts de Marketing Mix, de Personnel Mix (Martory et Croset, 1984), ou encore de Mix Social (Mahé de Boislandelle, 1988), il semblerait opportun d'envisager une réflexion en termes d'Emploi Mix, pour traduire cet arbitrage permanent entre les différentes composantes de la politique d'emploi de l'entreprise.

On pourrait ainsi définir l'Emploi Mix comme l'ensemble des variables dont l'entreprise dispose afin de réaliser un arbitrage entre ses effectifs et les besoins en ressources humaines nés de la stratégie.

Ainsi, diverses variables, pouvant être mises en œuvre de façon complémentaire ou bien alternative, s'offrent au dirigeant :

- en cas de besoins en ressources humaines : embaucher, promouvoir les ressources humaines existantes, externaliser ces ressources, ou encore précariser leur statut en faisant appel à des formes d'emploi particulières...;

- en cas d' excédents en ressources humaines : licenciement, arrêt de l'embauche, promotion des départs, arrêt momentané ou définitif des relations d'extemalisation,...

Bien entendu, les choix qui seront réalisés s'inscrivent dans le cadre d'une réflexion prévisionnelle compte tenu des objectifs de gestion des ressources humaines définis en osmose avec les orientations stratégiques de bases. La figure $n^{\circ} 3$, illustre le cadre d'ensemble dans lequel pourra s'effectuer la prise de décisions. 
Fig. 2 Les stratégies de base des P.M.E.

(Schéma réalisé à partir du modèle de M. Porter (1986))

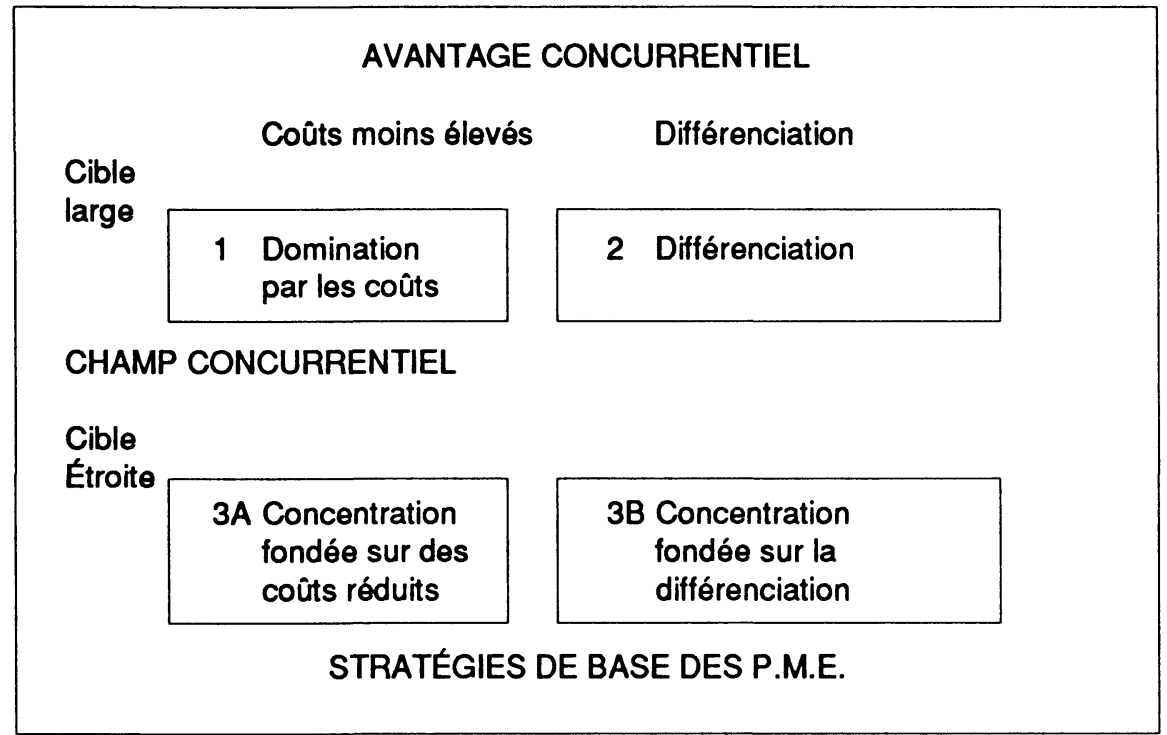

\subsection{Les politiques à mettre en œuvre}

Les politiques à mettre en œuvre dans le cadre d'une gestion stratégique de l'emploi dans les P.M.E. se doivent de prendre en considération deux groupes de contraintes et impératifs : en premier lieu, il importe que les choix opérés en matière de gestion de l'effectif soient cohérents avec les buts de l'organisation ou, ce qui revient au même, ceux de son dirigeant, voire de la famille dirigeante. A cette dimension organisationnelle, s'ajoute une dimension plus spécifiquement stratégique : le décideur de la politique de gestion de l'emploi devra intégrer celle-ci dans la stratégie globale de l'entreprise, compte-tenu des conditions d'incertitude de l'environnement.

\subsection{Pour une gestion de l'emploi compatible avec les buts dirigeants}

Nous savons que pour des raisons tout à fait respectables, certains dirigeants de P.M.E. ne souhaitent pas donner à leur affaire une ampleur trop importante. Certains limitent l'accroissement de leurs effectifs à des seuils à partir desquels ils considèrent que leurs aspirations fondamentales sont remises en question. Il va de soi que s'il est une variable sur laquelle on ne peut agir, c'est bien le "panier de buts» (Marchesnay, 1986) du dirigeant. Dès lors, se pose la question de savoir comment répondre aux opportunités de croissance qui se présentent à l'entreprise ? Il semble 
Fig. 3 La gestion des effectifs intégrée au processus de décision stratégique des PME

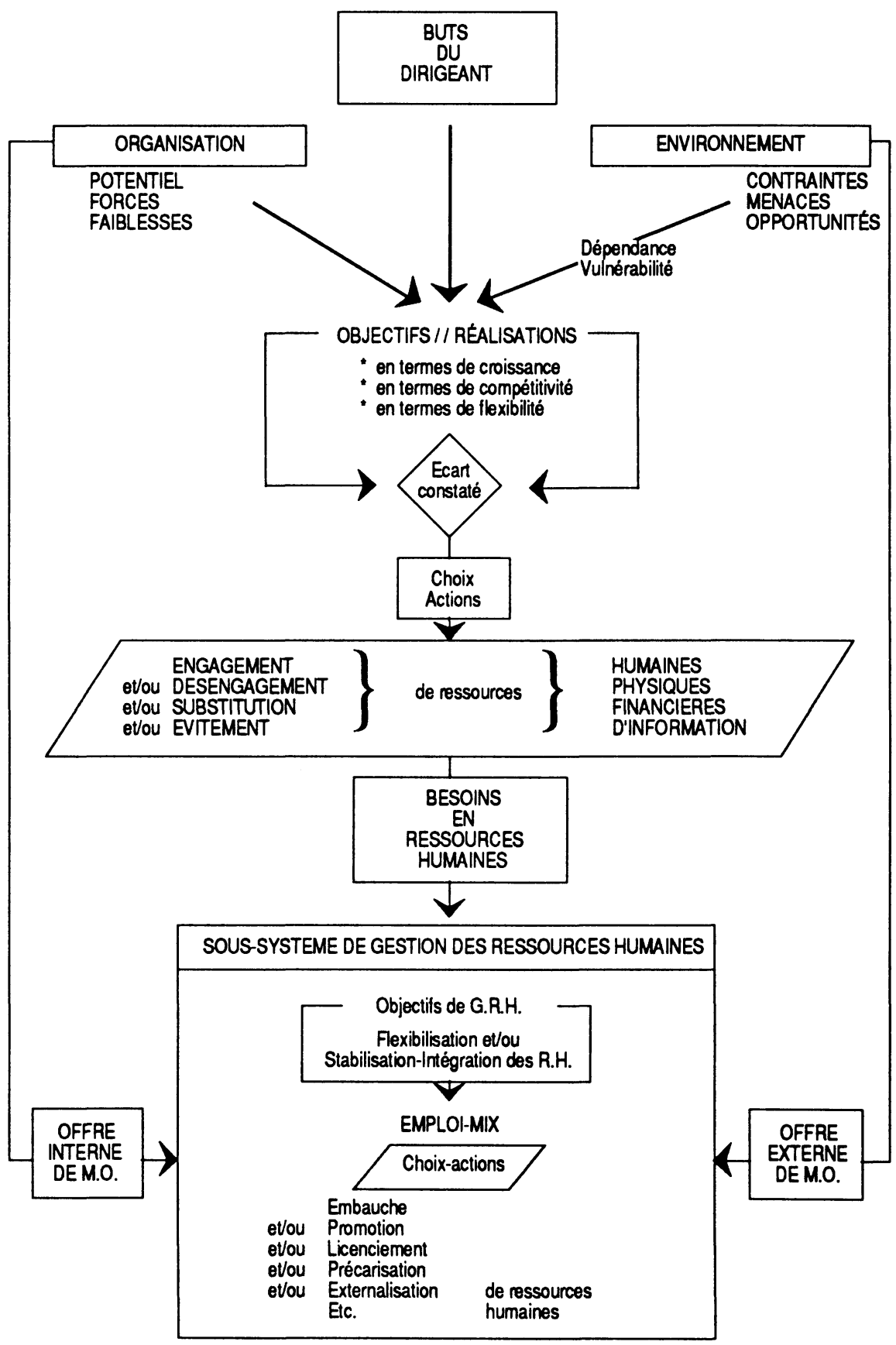


ici que la gestion de l'emploi doive s'orienter vers des pratiques d'évitement de l'embauche permettant à la P.M.E. de poursuivre sa croissance sans pour cela atteindre un effectif trop important. La question du recours à l'externalisation est donc envisageable dans ce cas. On pourra alors, pour des besoins ponctuels bien définis, recourir à une main d'œuvre d'appoint (intérimaires, salariés sous contrat à durée déterminée, travailleurs à domicile, etc.).

Citons à titre d'exemple le cas de ce créateur de cheminées métalliques qui faisait réaliser la fabrication de ses produits en sous-traitance et confiait la pose chez le client à des artisans implantés dans la localité où celle-ci devait s'effectuer. Pour des besoins ponctuels, notamment lors des opérations de finissage, le chef d'entreprise faisaitalors appel à un unique apprenti. Ainsi pouvait-il se consacrer pleinement et dans les meilleures conditions à la création de modèles.

De même, pour certains dirigeants ayant créé leur affaire afin de vivre dans leur métier des relations humaines conviviales au sein d'une petite équipe de travail, mais désirant malgré tout poursuivre la croissance des performances de l'entreprise, (c'est un cas très fréquent notamment chez les jeunes créateurs d'entreprise Blaquière et Bastide, 1987), l'externalisation d'activités, et donc d'emplois, pourrait être conseillée.

\subsection{Intégrer la gestion de l'emplol dans la stratégie globale de l'entreprise...}

Dans les entreprises recherchant leur avantage compétitif dans des coûts plus faibles que ceux de leurs concurrents, la main-d'œuvre peut être perçue comme une contrainte, voire comme un risque, qu'il convient de maitriser : diminuer la charge financière que constitue le personnel, accroître sa flexibilité, deviennent dès lors les impératifs de la gestion de l'emploi, ce qui implique des mesures adéquates : externalisation substitution du capital au travail, précarisation de la main-d'œuvre.

Dans les P.M.E. qui au contraire, fondent leur compétitivité sur la recherche d'un meilleur service à la clientèle, la main-d'œuvre devient alors une ressource qu'il conviendra de valoriser. Elle apparaît ainsi comme un élément essentiel du potentiel de compétitivité de l'entreprise. Cela se comprend d'autant mieux dans la mesure où ces firmes cherchent avant tout à favoriser l'interactivité avec leurs clients. Il importe dès lors de mettre au service de ces derniers un personnel motivé et capable de s'adapter à leurs besoins, et cela d'autant plus que ceux-ci seront complexes et changeants. Par ce moyen, l'entreprise pourra s'assurer une meilleure fidélité de sa clientèle et réduire ainsi une part de sa vulnérabilité commerciale et de l'incertitude qu'elle engendre. Les pratiques de gestion de l'emploi devront alors obéir à un impératif de stabilisation des ressources humaines (il importe en effet d'assurer un suivi, une permanence, voire une certaine personnalisation dans les relations avec la clientèle), ainsi qu'à un impératif d'intégration de personnel devant être suffisamment motivé au point de partager la mission de l'entreprise auprès de ses clients.). 
Il semble également possible d'admettre dans ce type de situation une recherche de flexibilité du personnel (celui-ci devant s'adapter en permanence aux exigences et aux besoins de la clientèle ainsi qu'à leur évolution), mais la différence essentielle avec le cas précédent, c'est que la flexibilité devra passer par d'autres moyens que celui de la précarisation de la main d'œuvre. On peut également concevoir dans ce cas d'entreprises recherchant leur compétitivité dans un meilleur service à la clientèle, des politiques d'externalisation, notamment pour des activités moins maîtrisées, afin de pouvoir se consacrer en exclusivité au métier et à la mission d'excellence de la firme. Le problème sera alors de rencontrer des sous-traitants ou des prestataires de services présentant tous les critères de qualité que réclament les orientations stratégiques. On peut aussi admettre une précarisation du statut de certains employés pour des besoins ponctuels n'affectant pas les activités génératrices de valeur (Porter, 1986); afin aussi, peut-être, de pouvoir assurer les conditions de stabilité des effectifs permanents. Enfin, et surtout, l'intégration d'un personnel polyvalent pourrait alors constituer un moyen d'obtenir la flexibilité des ressources humaines.

En fait, cette exigence de flexibilité ne réclame pas les mêmes solutions selon que la firme recherche un avantage de coût ou qu'à l'inverse, elle poursuive un objectif de compétitivité par le service, elle demeure largement contingente aux conditions d'environnement.

\subsection{Adapter la gestion de l'emplol}

En effet, quel que soit le type d'avantage compétitif recherché par la P.M.E., son dirigeant devra tenir compte des conditions d'environnement dans lesquelles se déroule son activité. Il importe dès lors de considérer le degré d'incertitude et de contrainte introduit par les conditions de dépendance et de vulnérabilité de la firme. Cela peut conduire notamment à adapter la gestion des effectifs en fonction de la nature des relations d'échange qui lient l'entreprise à ses partenaires : cela pose tout le problème de la gestion de l'emploi dans les P.M.E. sous-traitantes.

\section{Adapter la gestion de l'emploi aux conditions d'incertitude...}

La matrice illustrée dans la figure $n^{\circ} 4$ fournit diverses orientations concernant les objectifs vers lesquels devra se diriger la gestion de l'emploi en fonction, d'une part du type de stratégie adoptée en matière de compétitivité et, d'autre part, des conditions de l'environnement.

A la lecture de la matrice, quatre cas de figure sont envisageables.

Dans un premier cas, la P.M.E. fonde sa compétitivité sur des coûts plus faibles que ceux de ses concurrents et évolue dans un environnement marqué par une forte incertitude liée aux conditions de dépendance et de vulnérabilité qui caractérisent sa position stratégique. Dans ce cas, la flexibilité des ressources de l'entreprise 
Fig. 4 Une matrice stratégle/environnement dans le cadre d'une gestion stratégique de l'emploi dans les P.M.E.

\begin{tabular}{|c|l|l|}
\cline { 2 - 3 } \multicolumn{1}{c|}{} & \multicolumn{1}{c|}{ CARACTERISTIQUES DE L'ENVIRONNEMENT } \\
\hline $\begin{array}{c}\text { Orientations stratégiques } \\
\text { de base }\end{array}$ & \multicolumn{1}{|c|}{$\begin{array}{c}\text { Incertitude } \\
\text { élevée }\end{array}$} & \multicolumn{1}{c|}{$\begin{array}{c}\text { Incertitude } \\
\text { faible }\end{array}$} \\
$\begin{array}{l}\text { Avantage compétitif par } \\
\text { les coûts }\end{array}$ & $\begin{array}{l}\text { Flexibilisation par } \\
\text { précarisation et } \\
\text { évitement de la } \\
\text { main-d'œuvre }\end{array}$ & $\begin{array}{l}\text { Stabilisation d'un } \\
\text { effectif réduit aux } \\
\text { conditions précaires }\end{array}$ \\
\hline $\begin{array}{l}\text { Avantage compétitif par } \\
\text { les services }\end{array}$ & $\begin{array}{l}\text { Flexibilisation par } \\
\text { l'intégration d'un } \\
\text { personnel polyvalent }\end{array}$ & $\begin{array}{l}\text { Stabilisation et } \\
\text { intégration des } \\
\text { ressources } \\
\text { humaines }\end{array}$ \\
\hline
\end{tabular}

va s'ériger en impératif. En outre, la main-d'œuvre, en raison de son coût, est perçue comme une contrainte. Aussi, importera-t-il d'assurer sa flexibilité par des mesures de précarisation de l'emploi ainsi que par un évitement de capacités humaines (externalisation, augmentation de l'intensité capitalistique...) (Reix, 1979). Parmi les P.M.E. interrogées en Languedoc-Roussillon lors de nos diverses enquêtes, les moyennes entreprises oeuvrant dans le secteur de la sous-traitance électronique, ou encore dans la métallurgie et le textile pouvaient être classées dans ce cas.

Dans un second cas, des P.M.E. recherchant leur compétitivité dans un avantage de coût œuvrent dans un environnement stable caractérisé par une incertitude réduite. Dans ce cas, il importe davantage de réduire le coût de la main-d'œuvre que d'assurer sa flexibilité; aussi l'entreprise va-t-elle chercher à réduire sa masse salariale par la stabilisation d'un effectif réduit aux conditions précaires (en termes de salaires, de statut...). La politique devra s'orienter alors vers le recrutement d'une main-d'œuvre peu qualifiée, acceptant des conditions précaires, caractérisée par une ambition limitée. La stabilisation porte ici davantage sur le niveau de l'effectif que sur son statut. Les conditions de travail pourront cependant engendrer des taux de rotation élevés du personnel. La stabilisation du niveau de l'effectif et la réduction de son coût pourraient par ailleurs être obtenues par une politique de substitution de capacités techniques à des capacités humaines, mais également par un recours à l'externalisation de l'emploi (sous traitance, externalisation de services, travail à domicile, etc.) voire d'internationalisation (implantation dans des pays à maind'œuvre bon marché). A titre d'exemple, on peut citer le cas de certaines P.M.E. du secteur textile. Mentionnons également le cas de petites écoles privées qui, recherchant davantage la diminution de leurs coûts que la qualité de leurs prestations, font 
appel à une main-d'œuvre précaire, caractérisée par un roulement élevé (étudiants et vacataires divers,...).

Un troisième cas se caractérise par des P.M.E. qui fondent leur compétitivité sur la qualité de leurs prestations et qui évoluent dans un environnement marqué par une forte incertitude. Dans ce cas, même si cette incertitude peut être réduite par une fidélisation de la clientèle, le caractère évolutif et complexe des besoins de cette dernière nécessite une grande flexibilité des ressources humaines. Celles-ci deviennent dès lors un élément essentiel du potentiel de compétitivité. C'est en effet sur un personnel motivé, polyvalent et disponible que devra compter le dirigeant pour satisfaire les besoins de ses clients. La gestion de l'emploi devra alors s'orienter vers une politique de flexibilisation par l'intégration.

Divers exemples nous sont donnés par de petites entreprises du secteur électroniques, des S.S.C.I., ou encore par des entreprises oeuvrant dans des activités très différenciées (bonneterie de luxe par exemple).

Enfin, un quatrième cas regroupe des entreprises évoluant dans un environnement où l'incertitude est réduite et dont les activités sont fondées sur un meilleur service. Là, il s'agira avant toute chose de stabiliser la main-d'œuvre, voire de rechercher son intégration. Pour illustrer ce type de situation, nous pouvons citer le cas de petits commerces de détail, certains cabinets d'experts comptables, ou de petites entreprises agro-alimentaires différenciées sur un produit traditionnel de qualité.

\section{Adapter la gestion de l'emplol à la nature des relations d'échange : le cas particulier des P.M.E. sous-traltantes...}

Enfin, la gestion de l'emploi devra être adaptée aux conditions dans lesquelles se réalisent les échanges avec les partenaires, et notamment avec les donneurs d'ordre, dans le cas de firmes sous-traitantes, qu'il s'agisse de soustraitance de production ou de services. Deux cas sont envisageables : une situation de dépendance envers un donneur d'ordre qui recherche dans la relation des avantages de coût ou de flexibilité et tente de rejeter sur la P.M.E. une part de ces contraintes; une situation que l'on pourrait qualifier d'interdépendance, dans laquelle le client cherche davantage à favoriser un partenariat fondé sur la recherche commune de qualité.

\section{Gérer l'emploi en situation de dépendance...}

Dans un tel cas, la P.M.E. aura intérêt à son tour à rejeter une part de l'incertitude introduite par sa situation de dépendance sur d'autres entreprises par un recours à l'externalisation, ou bien en flexibilisant sa main-d'eouvre par la précarisation de son statut. 


\section{Gérer l'emploi en situation d'interdépendance...}

Ce type de relation peut caractériser une sous-traitance de spécialité où le donneur d'ordre recherche essentiellement dans ses sous-traitants la qualité des prestations fournies. Mais surtout, il peut s'agir de relations caractérisant un partenariat d'entreprises. Dès lors, la gestion de l'emploi devra s'orienter vers une politique d'intégration de personnel.

En dernière analyse, l'emploi apparaît bien comme une variable stratégique pour les firmes de petite et moyenne dimension et les décisions qui seront prises concernant l'engagement de ressources humaines exigent de la part de leur dirigeant la même attention que celle accordée à l'acquisition de resources techniques ou financières; tout nous porte à penser que c'est en considérant l'engagement de ressources humaines comme un investissement stratégique que l'on peut réaliser une gestion efficiente des effectifs dans les P.M.E

\section{Conclusion : l'avenir stratégique de la gestion des effectifs en P.M.E. : quelles conséquences sur les emplois de demain?}

Nous avons souligné qu'en fonction d'une part de la stratégie adoptée (compétitivité par les coûts ou par les services), et d'autre part, des caractéristiques de l'environnement concurrentiel, les objectifs de gestion de l'emploi devraient s'orienter dans des directions divergentes allant de la précarisation de l'emploi à sa stabilisation, voire à son intégration. Au delà de cette observation, se pose une interrogation fondamentale sur le devenir de la gestion de l'emploi et des ressources humaines en P.M.E. Cette question apparait d'autant plus cruciale, qu'elle a trait aux conditions mêmes dans lesquelles tend à se réaliser le rapport salarial et qu'elle affecte ainsi les conditions de travail offertes par ces entreprises; car derrière le phénomène de miniaturisation de l'économie (Delattre, 1984), c'est toute la question des emplois de demain qui se pose.

A ce propos, les conclusions de nos recherches laissent entrevoir deux visions qui, loin de s'opposer, sembleraient au contraire se juxtaposer.

Selon une première vision pessimiste, on assisterait à une aggravation du phénomène de l'augmentation du marché du travail. L'augmentation du nombre de P.M.E. dans l'économie aurait ainsi pour conséquence, un rapport salarial à plusieurs vitesses. Autour d'une population au travail stabilisée dans des grandes organisations, graviteraient un ensemble de salariés aux conditions précaires: personnel sous contrat à durée déterminée, intérimaires, travailleurs à domicile, mais aussi employés des firmes sous-traitantes ou prestataires de services. Ce scénario se rapprocherait fort de la situation japonaise, où coexistent un système d'emploi à vie, stabilisée dans les grands groupes industriels et un système d'emplois très précaires dans des P.M.E. 
sous-traitantes. En d'autres termes, l'éclatement du tissu productif en firmes de plus en plus petites conduirait à l'éclatement des statuts.

Dans une perspective plus optimiste, voire peut-être quelque peu idéaliste, l'évolution tendrait vers une nouvelle forme de rapport salarial caractérisé par la convivialité des relations de travail. Dans de petites structures conviviales, œuvrerait un personnel motivé et polyvalent auquel serait offert un élargissement des responsabilités. On retrouve ici le modèle de l' «ouvrier proudhonien» décrit par $R$. Boyer (1986). Ce modèle de petites entreprises flexibles se rapprocherait ainsi des observations faites par divers économistes. On peut citer à ce propos, les constatations faites par M. Piore et C. Sabel (1984) sur la situation italienne. Enfin, ce type de petites structures flexibles s'apparenterait au modèle d' «entreprises de troisième type», idéalisé par G. Archier et H. Serieyx (1984), dans lesquelles la performance serait recherchée dans l'adhésion du personnel aux objectifs de qualité et dans une capacité d'adaptation accrue de sa part. On rejoindrait ici les observations de T. Peters et R. Waterman (1983).

En réalité, au delà de ces deux scénarios extrêmes, on risque fort d'assister à une imbrication des situations. On peut en effet très bien concevoir, d'après nos propres observations en Languedoc-Roussillon, une coexistence entre d'une part des P.M.E. vulnérables, dépendantes, recherchant leur compétitivité, voire leur survie, dans des coûts de main-d'œuvre les plus bas possibles, adoptant des politiques de flexibilisation du personnel par la précarisation de son statut ou son externalisation et d'autre part, des P.M.E. plus protégées, notamment par une différenciation des produits ou une fidélisation de la clientèle, recherchant la stabilisation et l'intégration de leurs ressources humaines au sein d'une petite structure conviviale.

Au total, si elle fournit une approche nouvelle des processus qui régissent l'allocation des ressources humaines, la problématique de la gestion des effectifs dans les P.M.E. ouvre la voie à une réflexion sur la gestion sociale et les conditions de travail offertes par ces firmes appelées à devenir un lieu de travail qui tend désormais à occuper une place prépondérante dans la plupart des économies occidentales. Ainsi, par la richesse et la variété des interrogations qu'elles ne laissent pas de susciter, les P.M.E. et leur gestion des ressources humaines offrent aujourd'hui un terrain de recherche encore insuffisamment exploré, mais d'une fécondité extrêmement prometteuse.

1 Gérald Naro : «Facteurs et comportement d'embauche dans les P.M.E.», Thèse de doctorat de Science de Gestion, Université de Montpellier 1, février 1989.

Deux enquêtes furent réalisées dans le cadre de cette recherche : une première enquête qualitative sur les déterminants de l'embauche dans les PME, par entretiens semi-directifs auprès d'un échantillon de 50 chefs d'entreprise; une deuxième enquête sur les comportements d'embauche, à partir de questions fermées ayant donné lieu à une analyse de données, portant sur un échantillon de 257 dirigeants de P.M.E.

2 Ibidem 


\section{BIBLIOGRAPHIE}

Alfander, G., (1989), «Pour une stratégie des ressources humaines», Paris, Chotard et associés, $324 \mathrm{p}$.

Archier, G. et Serieyx, H., (1984), «L'entreprise du troisième type», Paris, Le Seuil, 222 p.

Besseyre Des Horts, C.H., (1988), «Vers une gestion stratégique des ressources humaines», Paris, Ed. d'Organisation, 234 p.

Birch, D., (1979), «The job generation process», Cambridge, Mass., M.I.T., program on neighbourhood and regional change.

Birch, D. et Mc Cracken, S., (1981), «Corporate evolution : a micro-based analysis», Cambridge, Mass., M.I.T. program on neighbourhood and regional change.

Birley, S., (1986), «Birth, death and job generation», Strategic Management Jouranl, vol. 7, p. 361-376.

Blaquière, B. et Bastide, B., (1987), «Comportements face à l'embauche dans les petites entreprises en démarrage», Montpellier, travaux de recherche du CEGERH.

Boyer, B., (1986), La flexibilité du travail en Europe, Paris, Ed. La Découverte, p. 237-283.

Delattre, M., (1984), «Le progrès des P.M.E. dans la crise», Communication au colloque de Trois-Rivières : «Les P.M.E. dans un monde en mutation», Trois-Rivières.

Didier, M., (1982), «Crise et concentration du système productif», Economie et Statistiques, $n^{\circ} 144$, mai 1982.

Faure, R., Marchesnay, M. et Mathie, B., (1979), «A l'ombre des grands», Revue Française de Gestion sept-oct. 1979, p. 108-115.

Gauthier, D. et Pelisse, H., (1985), «Organisation de la fonction personnel et taille des entreprises», Montpellier, Travaux de recherche du CEGERH.

Julien, P.A., Marenne, J. et Naro, G., (1988), «La place de la petite entreprise dans les économies en mutation», dans La petite entreprise, Paris, ouvrage collectif sous la direction de P.A. Julien et M. Marchesnay, Paris, Ed. Vuibert, p. 39-48.

Laroche, G., (1989), Petites et moyennes entreprises au Québec : organisation économique, croissance de l'emploi et qualité du travail, Genève, Institut International d'Études Sociales, 70 p.

Mahé de Boislandelle, H., (1988), Gestion des ressources humaines dans les P.M.E., Paris, Ed. Economica, 322 p.

Marchesnay, M., (1980), Stratégie, Paris, Ed. Chotard et associés, p. 58.

Marchesnay., M., (1988), «Stratégie des petites et moyennes entreprises», contribution au Traité d'économie industrielle, Paris, Economica.

Martory., B. et Crozet, D., (1984), Gestion des Ressources Humaines, Paris, Ed. Fernand Nathan, $255 \mathrm{p}$.

Miller, R., (1985), «Une politique de ressources humaines au service de la stratégie», Revue Française de Gestion, mars-avril-mai, 1985, p. 57-67.

Peters, T. et Waterman, R., (1983), Le prix de l' excellence, Paris, InterEditions, 1988, 359 p.

Piore, M. et Sabel, C., The Second Industrial Divide, New-York, Basic Books, 355 p.

Porter, M.E., (1986), L'avantage concurrentiel, Paris, InterEditions, 647 p.

Quin Mills., D., (1986), «Stratèges, n'oubliez pas les ressources humaines!», Harvardl'Expansion, printemps 1986, p. 32-46.

Reix, R., (1979), La flexibilité de l'entreprise, Paris, Ed. Cujas, p. 99.

Storey, D.J. et S. Johnson, (1986), «Job generation in Britain : a review of recent studies», International Small Business Journal, vol. 4, $\mathrm{n}^{\circ} 4$, été 1986, p. 29-46.

Storey, D.J. et Robinson, S., (1980), «Employment change in CLEVELAND 1966-1976», Centre for Environnemental Studies Research. 